Research Article

\title{
Phenotypic and Genotypic Detection of Biofilm Formation Pseudomonas oryzihabitance and Susceptibility to Antibiotics
}

\author{
Shaimaa Obaid Hasson \\ Al-Qasim Green University, Veterinary Collage, Department of Microbiology, Iraq. \\ _Corresponding author. E-mail: shaimaaobead@gmail.com Tel.: + 009647806202870
}

Received: Aug. 24, 2018; Accepted: Oct. 24, 2018; Published: Jan. 17, 2019.

Citation: Shaimaa Obaid Hasson, Phenotypic and Genotypic Detection of Biofilm Formation Pseudomonas oryzihabitance and Susceptibility to Antibiotics. Nano Biomed. Eng., 2019, I I ( I): I I- I7.

DOI: 10.5101/nbe.v11i1.p11-17.

\begin{abstract}
Pseudomonas oryzihabitans is uncommon pathogen, but recently there may be warning of this bacterium to be dangerous, highly virulence, and may cause increase in morbidity and mortality rate, especially if there are multi-drug resistance and biofilm former. Biofilm forming bacteria display resistance to antibiotics, reaching to 1000 time higher than planktonic bacteria. The aim of the current study was to detect Pseudomonas oryzihabitans as biofilm producer and determine the susceptibility to the tested antibiotics. Pseudomonas oryzihabitans was isolated from urine of catheterized patients in Iraqi hospitals and diagnosed as biofilm former phenotypically by congo red and tissue culture plate methods, and genetically by polymerase chain reaction analysis to detect las $R$ gene (quarm sensing gene) as biofilm indicator and determine its susceptibility to antibiotics by disc diffusion and VITEK AST method. The results revealed that Pseudomonas oryzihabitans was present at 3 isolates; all of them had lasR gene and were resistant to most tested antibiotics at $100 \%$, except that it showed sensitivity rate reaching to $100 \%$ to imipenem. From the present study, it was concluded that Pseudomonas oryzihabitans was isolated for the first time as biofilm producer from urinary catheter which possessed lasR gene as biofilm formation indicator, and the multi-drug resistance was considered as a distinctive feature of biofilm formation bacteria.
\end{abstract}

Keywords: Pseudomonas oryzihabitans; Biofilm; Multi-drug resistance; Antibiotics; Urinary catheter

\section{Introduction}

Pseudomonas oryzihabitans (P. oryzihabitans) is a gram negative yellow pigmented, oxidase negative, rod shaped bacteria. It is an opportunistic human pathogen may increase morbidity and mortality rate by causing infection such as septicemia, bacteremia, peritonitis and endophthalmitis [1], but it is an uncommon pathogen and usually causes infection to immune compromised patients [2]. Some research pointed out an association between the bacteria and a catheter present in their patient's body [3] which was isolated for the first time from urine specimen [4, 5]. Recently, there are awareness and alert of pathogenic role of $P$. oryzihabitans which may increase virulence in future [5].

Most gram negative bacteria are prevalent as nosocomial infection related with urinary tract 
infection (UTI) and are also associated with catheter (CAUTI) $[6,7]$. Among the gram negative bacteria that are associated with biofilm infections, Pseudomonas aeruginosa has attracted more attention [8]. In general, Pseudomonas sp. are a causative agent for many infections such as UTI, endocarditis, septicemia etc., and mainly have challenge to act as multi-drug resistant bacteria at alarming rate $[9,10]$.

Pseudomonas sp. are characterized by having the ability of biofilm formation and multi-drug resistance at highly level [11]. They have the ability to produce urease enzyme which permits it to form crystalline biofilm associated with long indwelling urinary catheter leading to block the catheter [12].

In the last years, studies about biofilm have grown obviously due to its seriousness role on public health. Biofilm formation in urinary catheter causes kidney failure that leads to death, as mass biofilm prevents urine passing through the catheter and returning back to the kidney [13]. In addition to the role of biofilm, there is a high incidence rate of antibiotic resistance.

In general, biofilm formation goes in three major stages: (i) attachment to a selected surface; (ii) colonization and growing up to be mature structure of biofilm; (iii) detachment or called dispersal. Within bacterial biofilm, each bacterial cell communicates with each other via signal small chemical molecules auto-inducers called quarm sensing (QS) which exist in gram negative acyl-homoserin lactones (AHL) [14]. Then, bacterial cell starts to aggregate to each other through cell to cell interaction, and then produces a matrix of extracellular polymeric substance (EPS) which is composed of polysaccharides, lipid, protein and extracellular DNA (eDNA) [13, 15]. This matrix encapsulates bacteria to act as protective layer from undesired environmental conditions such as antibiotics action, biocides, dryness, U.V radiation, stress factors and defense of host immune system [16].

Because there hasn't been any study about biofilm formation of $P$. oryzihabitans in urinary catheter, the aim of the current study was to determine the biofilm-forming capacity of $P$. oryzihabitans isolated from urinary tract catheterized patients and evaluate Pseudomonas biofilm gene lasR (gene encoding for quarm sensing) in biofilm producing $P$. oryzihabitans for the first time.

\section{Experimental Collection of samples}

Sixty-five of $5 \mathrm{~mL}$ urine samples were collected from catheterized patients in sterile test tubes and transported immediately to laboratory. The patients at different age groups and to both sex were admitted to different departments of some Iraqi hospitals during the period from February to April 2017, and all the patients had been exposed to antibiotics for at least 3 days.

\section{Identification of bacterial isolates}

Urine samples were cultured in enrichment media (brain heart infusion broth (Oxoid, UK) and incubated at $37{ }^{\circ} \mathrm{C}$ for $24 \mathrm{~h}$, then cultured in routinely media for bacterial growth (nutrient, macconky and blood agar (oxoid, UK) and incubated at $37{ }^{\circ} \mathrm{C}$ for $24 \mathrm{~h}$. Bacterial culture growth was diagnosed by biochemical test, staining and microscopic examination $[17,18]$. Then bacterial isolates were identified by the automated system VITEK 2 (bioMerieux, Marcy I'Etoile, France) to reach final diagnosis and identification.

The identification with VITEK 2 included IDGN card for gram-negative enterobactericeae. P. aeruginosa was used as reference bacterial strain (control) which was diagnosed according to the method above.

\section{Biofilm detection}

The biofilm was detected by three methods:

\section{Congo red agar method}

The method was done according to Freeman et al. [19]. The result was as follows: If the bacteria formed black colonies with a dry crystalline consistency, it means the bacteria were biofilm producer strains, and if it formed red colonies, it means the bacteria were non-biofilm producer strains [20].

\section{Tissue culture plate (TCP) method}

The method was done according to Christensen et al. [21]. The mean of absorbance value from replicate wells was read, and the biofilm degree was calculated according to this equation: Biofilm degree $=$ Mean $\mathrm{OD}_{630}$ of tested bacteria - Mean $\mathrm{OD}_{630}$ of control. The results were interpreted according to Table 1. Modified TCP method was considered as gold standard [22].

\section{Polymerase chain reaction (PCR) method}

The study on $P$. oryzihabitans biofilm formation gene (lasR) was performed by PCR technique. The primer was designed in this study by using National Center for Biotechnology Information (NCBI) gene sequence data base and primer 3 plus design. This 
Table 1 Classification based on optical density (OD) values

\begin{tabular}{ccc}
\hline Mean OD value & Adherence & Biofilm formation \\
\hline$<0.120$ & Non & Non $/$ Weak \\
-0.240 & Moderate & Moderate \\
$>0.240$ & Strong & Strong \\
\hline
\end{tabular}

Table 2 PCR primers and their sequence

\begin{tabular}{cccc}
\hline Primer & & Sequences $(5 \rightarrow 3)$ & Amplicon \\
\hline \multirow{2}{*}{ lasR gene } & $\mathrm{R}$ & GGCTGTGTTCTCTCGTGTGA & $242 \mathrm{bp}$ \\
& $\mathrm{F}$ & CCTTGAACACTTGAGCACGC & \\
\hline
\end{tabular}

Note: Genbank: lasR: AM778435.1; F: forward; R: reversed

primer was provided by Bioneer, South Korea (Table 2).

\section{DNA extraction}

DNA extraction was done by treatment of bacterial isolates with DNA extraction kit according to manufacture protocol (Presto Mini-DNA Bacteria Kit. Geneaid Biotech Ltd. USA). Then, extracted DNA was estimated by nanodrop device at 260/280 $\mathrm{nm}$.

Master mix preparation

The mix was prepared using master mix reagents according to instruction of Accu- Power ${ }^{\circledR P C R}$ PreMix-Kit master mix reagent in a $20 \mu \mathrm{L}$ reaction mixture containing $5 \mu \mathrm{L}$ DNA template, $1.5 \mu \mathrm{L}$ of each primer and $12 \mu \mathrm{L}$ PCR water. The mixture was placed in AccuPower PCR-PreMix that contained all PCR components (Taq DNA polymerase, dNTPs, and 10 PCR buffers). Then, all the PCR tubes were transferred into vortex vibration for 3 min and transferred into thermocycler apparatus (MyGene, Bioneer. Korea).

PCR thermocycler conditions

The amplification was performed as follows: Initial denaturation step at $94{ }^{\circ} \mathrm{C}$ for $3 \mathrm{~min}(1$ cycle), followed by denaturation at $94{ }^{\circ} \mathrm{C}$ for $30 \mathrm{sec}$, annealing at 58 ${ }^{\circ} \mathrm{C}$ for $30 \mathrm{sec}$, extension at $72{ }^{\circ} \mathrm{C}$ for $1 \mathrm{~min}$, all for 30 cycles and final extension at $72{ }^{\circ} \mathrm{C}$ for $5 \mathrm{~min}$ ( 1 cycle) and holding $4{ }^{\circ} \mathrm{C}$ forever.

PCR product analysis

The PCR products were visualized by electrophoresis apparatus in a $1 \%$ agarose substance by using buffer, after staining with ethidium bromide, and observed under the ultraviolet (UV) transilluminator.

\section{Antimicrobial susceptibility testing}

The test was carried out by the following two methods.

\section{Disc diffusion method [23]}

The test was done on muller hinton agar with antibiotics disc containing, amikacin $30 \mu \mathrm{g}$, azithromycin $15 \mu \mathrm{g}$, carbencillin $100 \mu \mathrm{g}$, clindamycin $2 \mu \mathrm{g}$, imipenem $10 \mu \mathrm{g}$, penicillin G $10 \mathrm{IU}$ and tobramycin $10 \mu \mathrm{g}$ from Bioanalysis, India.

\section{MIC by VITEK system antibiotic sensitivity test (AST)}

The test was done according to VITEK instruction to multi-antibiotics, including ampicillin/calvulanic, cefazolin, ceftazidime, ceftriaxone, cefepime, ertapenem, imipenem, gentamicin, tobramycin, ciprofloxacin, levofloxacin, nitrofurantoin and trimetheprium/ sulfamithaxzol, by using ID card for enterobactericeae AST.

Results of the two methods were read as sensitive, intermediate or resistant according to the Clinical and Laboratory Science Institute (CLSI) standard guidelines, M100, 2017 [24].

\section{Statistical analysis}

All experiments were carried out in triplicate to validate the reproducibility of the experiments. Statistical analysis was done by using one way ANOVA at p-value 0.05 by SPSS Statistics 24.0 software.

\section{Results and Discussion}

The results revealed (Fig. 1) that among 28 biofilm bacterial isolates, $3(11 \%) P$. oryzihabitans which were isolated at the first time as biofilm producer from urinary catheterized patients and were alarmed to be dangerous bacteria. Some research pointed out that $P$. oryzihabitans may persist biofilm formation [2].

Moreover, other Pseudomonas species reported previously had been isolated from urinary catheter as strongly biofilm former [25]. They had the ability to

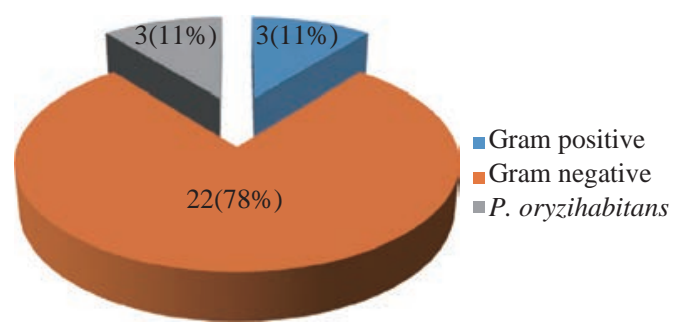

Fig. 1 Number and percentage of biofilm formation $P$. oryzihabitans isolated among other biofilm formation bacterial isolates from urinary catheter. 
produce urease enzyme which permitted them to form crystalline biofilm associated with long indwelling urinary catheter, leading to blocking the catheter [12]. Urease enzyme hydrolyzed urea to ammonia, elevated ammonia concentration, and led to the rise of urinary $\mathrm{pH}$, resulting in urinary salt precipitation to form kidney and bladder stones [26]. Urinary salts calcium and magnesium ammonium phosphate, at precipitation, incorporated with microbial polysaccharide to form crystalline biofilm on catheter surface [27].

\section{Detection of biofilm formation}

Biofilm formation of $P$. oryzihabitans was detected by three methods. In congo red method, $P$. oryzihabitans appeared as black colony, which was related to the stain of polysaccharide matrix formation by congo red stain [22] (Fig. 2). On the other hand. TCP method revealed that $P$. oryzihabitans displayed moderate ability to form biofilm in the range of 0.125 $0.240 \mathrm{~nm}$ with no significant difference between isolates as shown in Table 3.

\section{Molecular assay}

The biofilm formation process was controlled at the genetic level and by environmental signals regulation.

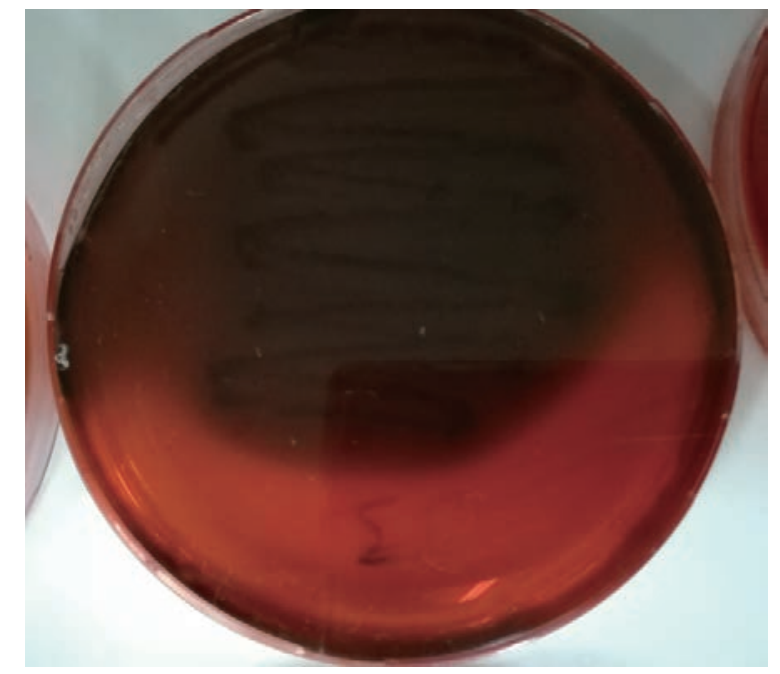

Fig. 2 Congo red agar indicating the biofilm of P. oryzihabitans.

Table 3 Optical density (OD) value of $P$. oryzihabitans biofilm degree according to TCP method

\begin{tabular}{ccc}
\hline Bacteria & Optical density $(630 \mathrm{~nm})$ & Biofilm degree \\
\hline P. oryzihabitans 1 & $0.125 \pm 0$ & Moderate \\
& $\mathrm{A}$ & \\
P. oryzihabitans 2 & $0.200 \pm 0$ & Moderate \\
& $\mathrm{A}$ & \\
P. oryzihabitans 3 & $0.230 \pm 0$ & Moderate \\
\hline
\end{tabular}

Note: Values represent mean \pm SE; similar capital letters mean no significant differences $(p \geq 0.01)$ between different optical densities.

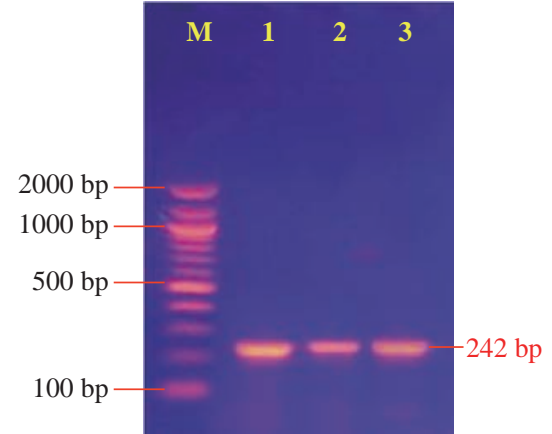

Fig. 3 Agarose gel electrophoresis of PCR assay showing the same positive results of biofilm formation lasR gene in Pseudomonas sp. positive isolates. Lane (M) DNA marker (1002000 bp); Lane (1-3) Positive lasR at 242 bp PCR product size.

PCR analysis results of lasR gene which were encoded for QS in P. oryzihabitans isolates revealed that all $3 P$. oryzihabitans isolates had this gene (Fig. 3), explaining their ability to form biofilm.

The important aspect of Pseudomonas sp. is its ability of environmental condition adaptation by transcriptional regulation of virulence factor genes such as QS gene which encodes communication of cell to cell to form biofilm [28]. And the transcriptional regulation of these genes was controlled by two QS systems: Last/R and RhlI/R [29].

Most gram negative bacteria expressed genetically QS signaling system, which means that N-acyl-1homoserine lactones (AHLs) influence many aspect of biofilm formation including motility, adhesion, maturation and dispersion [30, 31], and it's widespread in gram-negative bacteria, especially having been described for pseudomonas oryzihabitans. Many genes are responsible for expressing the product of QS and regulating its process in their biofilm bacteria on urinary catheter [32], such as lasR gene in $P$. oryzihabitans.

lasR has two domains, the $\mathrm{N}$ - terminal binding to auto-inducer binding domain and $\mathrm{C}$ - terminal binding to DNA [33]. The activity of this protein was regulated by auto-inducer ligand $\mathrm{N}$-(3-oxododecanoyl)homoserine lactone (3-oxo-C12-HSL) which binds and activates the cognate response regulator and exerts its transcriptional regulatory activity [28, 34]. That is one of the QS systems which are present in Pseudomonas sp. and considered as pathogenicity indicator.

\section{Antibiotic susceptibility testing}

The test was evaluated by two methods, disc diffusion and VITEK AST methods, which were determined according to CLSI [24]. The results 


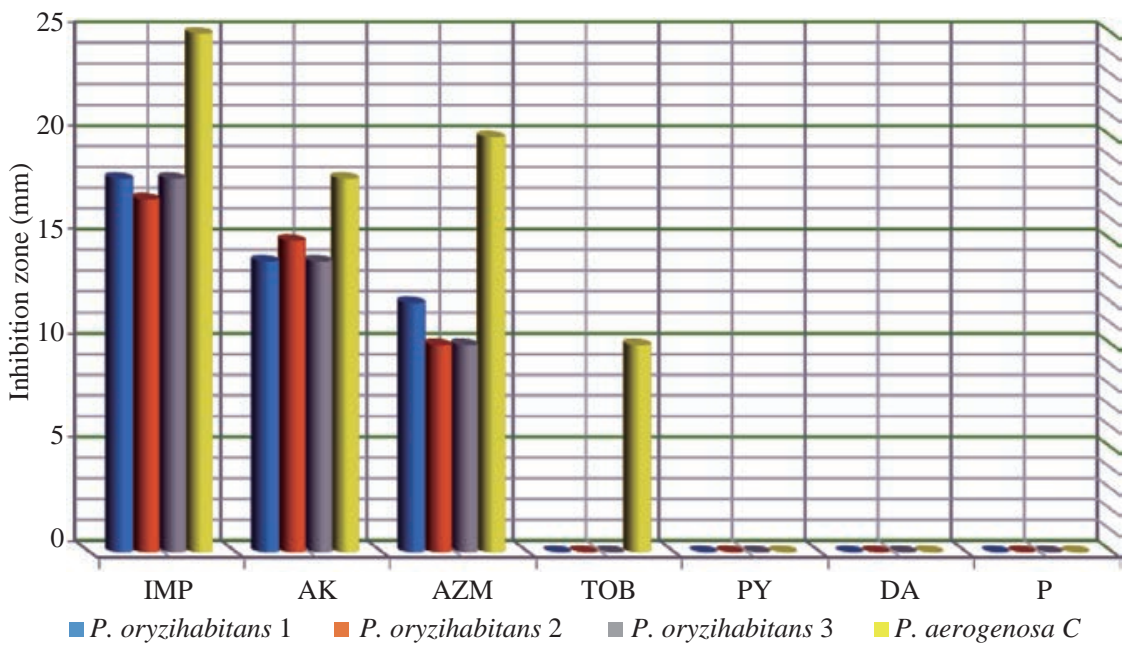

Fig. 4 Zone of inhibition (mm) of different antibiotics in disc diffusion method against P. oryzihabitans and P. aeruginosa as control. (IMP = imipenem; $\mathrm{AK}=$ amikacin; $\mathrm{AZM}=$ azithromycin; $\mathrm{TOB}=$ tobramycin; $\mathrm{PY}=$ carbenicillin; $\mathrm{DA}=$ clindamycin; $\mathrm{P}=$ penicillin.$)$

discovered that biofilm formation P. oryzihabitans was resisted to most tested antibiotics (Fig. 4) at $100 \%$ to amikacin, azithromycin, tobramycin, clindamycin, carbencillin and penicillin, except imipenem which showed high activity against tested biofilm bacteria at $100 \%$ sensitivity rate.

The results above were confirmative by VITEK AST system as showed in Table 4 that biofilm formation $P$. oryzihabitans was resisted to all experimental antibiotics except imipenem with intermediate effect. So biofilm formation P. oryzihabitance was considered multi-drug resistant bacteria (MDR); the term MDR is granted to any reported bacterial isolate resisting at least 3 different antimicrobial classes [35]. These results clarified that biofilm formation bacteria could not be eliminated or treated by traditional antibiotics, which was related to many reasons, including block

Table 4 VITEK AST results of biofilm forming P. oryzihabitans

\begin{tabular}{ccc}
\hline \multirow{2}{*}{ Antibiotics } & \multicolumn{2}{c}{ Pseudomonas } \\
\cline { 2 - 3 } & MIC & Interp. \\
\hline Ampicillin & $\geq 32$ & $\mathrm{R}$ \\
Ampicillin/clavulanic & $\geq 32$ & $\mathrm{R}$ \\
Ampicillin/sulbactaum & $\geq 32$ & $\mathrm{R}$ \\
Cefazolin & $\geq 64$ & $\mathrm{R}$ \\
Ceftazidime & $\geq 64$ & $\mathrm{R}$ \\
Ceftriaxone & $\geq 64$ & $\mathrm{R}$ \\
Impienem & 8 & $\mathrm{I}$ \\
Gentamicin & $\geq 16$ & $\mathrm{R}$ \\
Tobramycin & $\geq 16$ & $\mathrm{R}$ \\
Ciprofloxacin & $\geq 4$ & $\mathrm{R}$ \\
Levofloxacin & $\geq 8$ & $\mathrm{R}$ \\
Nitrofurantain & $\geq 512$ & $\mathrm{R}$ \\
Trimetheprium/sulfamithaxzol & $\geq 320$ & $\mathrm{R}$ \\
\hline
\end{tabular}

antibiotics penetration through multilayers matrix, modification of antibiotics by bacterial enzymes, and efflux pump to some antibiotics [36]. Extracellular polysaccharide matrix (EPS) was reported to protect Pseudomonas from tobramycin activity [37]. Waste production as a result of high density of biofilm may affect the action of antibiotics [38]. In addition to microenvironment represented by low nutrients, oxygen and chemical gradients which lead to low metabolic activity and decrease in cell division rate could result in slow growth or no growth of convert activity bacterial cell to semi dormant cell [39, 40]. Also, Pseudomonas bacterial isolates had the ability to form priplasmic glucan which sequestered antibiotic molecules and prevented the antibiotic reaching to its target [41]. All of these contribute to the resistance of bacterial cells in biofilm against antibiotics.

Antibiotic resistance patterns in biofilm forming pathogens were a protective mode to avoid antimicrobial action of traditional antibiotics that aid to persist infection in UTI

Imipenem has a broad spectrum effect on nosocomial Pseudomonas as anti- pseudomonal $\beta$ lactam antibiotic, in addition to its good activity against Pseudomonas sp., it has important role in treatment of severe infection and complicated UTI [42].

\section{Conclusions}

From the present study, it was concluded that Pseudomonas oryzihabitans was isolated for the first time as biofilm producer from urinary catheter which 
possessed lasR gene as biofilm formation indicator. The multi-drug resistance was considered as distinctive feature of biofilm formation bacteria. Imipenem was a highly effective antibiotic against biofilm bacteria.

\section{References}

[1] J. Freney, W. Hansen, J. Etienne, et al., Postoperative infant septicemia caused by Pseudomonas luteola (CDC group Ve-1) and Pseudomonas oryzihabitans (CDC group Ve-2). Journal of Clinical Microbiology, 1988, 26(6): 1241-1243.

[2] L. Dussart, J.P. Dupont, I. Zimmerlin, et al., Occurrence of sessile Pseudomonas oryzihabitans from a karstified chalk aquifer. Water Research, 2003, 37(7): 1593-1600.

[3] F. Lejbkowicz, L. Belavsky, R. Kudinsky, et al., Bacteraemia and sinusitis due to Flavimonas oryzihabitans infection. Scandinavian Journal of Infectious Diseases, 2003, 35(6-7): 411-413.

[4] K.G. Lucas, T.E. Kiehn, K.A. Sobeck, et al., Sepsis caused by Flavimonas oryzihabitans. Medicine, 1994, 73(4): 209-214.

[5] S.M. Bhatawadekar, Community-acquired urinary tract infection by Pseudomonas oryzihabitans. Journal of Global Infectious Diseases, 2013, 5(2): 82.

[6] S.M. Jacobsen, D.J. Stickler, H.L. Mobley, et al., Complicated catheter-associated urinary tract infections due to Escherichia coli and Proteus mirabilis. Clinical Microbiology Reviews, 2008, 21(1): 26-59.

[7] J.R. Johnson, M.A. Kuskowski, and T.J. Wilt, Systematic review: antimicrobial urinary catheters to prevent catheter-associated urinary tract infection in hospitalized patients. Annals of Internal Medicine, 2006, 144(2): 116126.

[8] H.S. Joo, M. Otto, Molecular basis of in vivo biofilm formation by bacterial pathogens. Chemistry \& Biology, 2012, 19(12): 1503-1513.

[9] N.K.K. Hindi, S.O. Hasson, and S.K.K. Hindi, Bacteriological study of urinary tract infections with antibiotics susceptibility to bacterial isolates among honeymoon women in Al Qassim Hospital, Babylon Province, Iraq. British Biotechnology Journal, 2013, 3(3): 332-340.

[10] N.K. Palanisamy, N. Ferina, A.N. Amirulhusni, et al., Antibiofilm properties of chemically synthesized silver nanoparticles found against Pseudomonas aeruginosa. Journal of nanobiotechnology, 2014, 12(1): 2.

[11] L. Zhang, M. Fritsch, L. Hammond, et al., Identification of genes involved in Pseudomonas aeruginosa biofilmspecific resistance to antibiotics. PLoS One, 2013, 8(4): e61625.

[12] J.K. Hatt, P.N. Rather, Role of bacterial biofilms in urinary tract infections. Curr Top Microbiol Immunol, 2008, 322: 136-192.

[13] C. Delcaru, I. Alexandru, P. Podgoreanu, et al., Microbial biofilms in urinary tract infections and prostatitis: etiology, pathogenicity, and combating strategies. Pathogens, 2016, 5(4): 65.

[14] M. Chifiriuc, G. Mihăescu, and V. Lazăr, Medical microbiology and virology. Univ. of Bucharest Publ. House, 2011.

[15] S. Neethirajan, M.A. Clond, and A. Vogt, Medical biofilms-nanotechnology approaches. Journal of Biomedical Nanotechnology, 2014, 10(10): 2806-2827.

[16] C. Limban, M.C. Chifiriuc, and A.M. Grumezescu, Thiourea derivatives as antimicrobials: Synthesis, biological activity and potentiation by nanotechnological solutions. LAP Lambert Academic Publishing, 2013.

[17] J.F. MacFaddin, Biochemical tests for identification of medical bacteria. 3rd ed. Williams and Wilkins. Baltimore, USA, 2000.

[18] P. Tille, Bailey \& Scott's Diagnostic Microbiology-EBook. Elsevier Health Sciences, 2015.

[19] D. Freeman, F. Falkiner, and C. Keane, New method for detecting slime production by coagulase negative staphylococci. Journal of Clinical Pathology, 1989, 42(8): 872-874.

[20] T.D. Kaiser, E.M. Pereira, K.R. Dos Santos, et al., Modification of the Congo red agar method to detect biofilm production by Staphylococcus epidermidis. Diagnostic Microbiology and Infectious Disease, 2013, 75(3): 235-239.

[21] G.D. Christensen, W.A. Simpson, J.J. Younger, et al., Adherence of coagulase-negative staphylococci to plastic tissue culture plates: a quantitative model for the adherence of staphylococci to medical devices. Journal of Clinical Microbiology, 1985, 22(6): 996-1006.

[22] S. Bose, M. Khodke, S. Basak, et al., Detection of biofilm producing staphylococci: Need of the hour. Journal of Clinical and Diagnostic Research, 2009, 3(6): 1915-1920.

[23] CLSI, Performance standards for antimicrobial disk susceptibility tests; Approved standard M02/ A12. $12^{\text {th }}$ ed. Clinical and Laboratory Standards Institute, Wayne, PA, 2015.

[24] CLSI, Performance standards for antimicrobial susceptibility testing. M100. Clinical and Laboratory Standards Institute, Wayne, PA., 2017.

[25] R.M. Donlan, Biofilms: microbial life on surfaces. Emerging Infectious Diseases, 2002, 8(9): 881.

[26] J.H. Tabibian, J. Gornbein, A. Heidari, et al., Uropathogens and host characteristics. Journal of Clinical Microbiology, 2008, 46(12): 3980-3986.

[27] A.L. Flores-Mireles, J.S. Pinkner, M.G. Caparon, et al., EbpA vaccine antibodies block binding of Enterococcus faecalis to fibrinogen to prevent catheter-associated bladder infection in mice. Science Translational Medicine, 2014, 6(254): 254ra127-254ra127.

[28] L. Steindler, I. Bertani, L. De Sordi, et al., LasI/R and RhlI/R quorum sensing in a strain of Pseudomonas aeruginosa beneficial to plants. Applied and Environmental Microbiology, 2009, 75(15): 5131-5140.

[29] R.S. Smith, B.H. Iglewski, P. aeruginosa quorum-sensing systems and virulence. Current Opinion in Microbiology, 2003, 6(1): 56-60.

[30] M.D. Koutsoudis, D. Tsaltas, T.D. Minogue, et al., Quorum-sensing regulation governs bacterial adhesion, biofilm development, and host colonization in Pantoea stewartii subspecies stewartii. Proceedings of the National Academy of Sciences, 2006, 103(15): 5983-5988.

[31] W.S. Tan, N.Y. Muhamad Yunos, P.W. Tan, et al., Pantoea sp. isolated from tropical fresh water exhibiting N-acyl homoserine lactone production. The Scientific World Journal, 2014, 2014: 828971.

[32] B.W. Trautner, R.O. Darouiche, Role of biofilm in catheter-associated urinary tract infection. American Journal of Infection Control, 2004, 32(3): 177-183.

[33] M.J. Bottomley, E. Muraglia, R. Bazzo, et al., Molecular insights into quorum sensing in the human pathogen $\mathrm{P}$. aeruginosa from the structure of the virulence regulator LasR bound to its autoinducer. Journal of Biological Chemistry, 2007, 282(18):13592-13600.

[34] M. Schuster, M. Urbanowski, and E. Greenberg, Promoter specificity in Pseudomonas aeruginosa quorum sensing revealed by DNA binding of purified LasR. Proceedings of the National Academy of Sciences, 2004, 101(45): 15833-15839.

[35] Z. Ghanbarzadeh Corehtash, A. Khorshidi, F. Firoozeh, 
et al., Biofilm formation and virulence factors among Pseudomonas aeruginosa isolated from burn patients. Jundishapur Journal of Microbiology, 2015, 8(10): e22345..

[36] M. Burmølle, D. Ren, T. Bjarnsholt, et al., Interactions in multispecies biofilms: Do they actually matter? Trends in Microbiology, 2014, 22(2): 84-91.

[37] R.D. Monds, G.A. O'Toole, The developmental model of microbial biofilms: Ten years of a paradigm up for review. Trends in Microbiology, 2009, 17(2): 73-87.

[38] J.D. Pozo, R. Patel, The challenge of treating biofilm associated bacterial infections. Clinical Pharmacology \& Therapeutics, 2007, 82(2): 204-209.

[39] K. Lewis, Persister cells and the riddle of biofilm survival. Biochemistry (Moscow), 2005, 70(2): 267-274.

[40] H. Van Acker, P. Van Dijck, and T. Coenye, Molecular mechanisms of antimicrobial tolerance and resistance in bacterial and fungal biofilms. Trends in Microbiology, 2014, 22(6): 326-333.

[41] T. Beaudoin, L. Zhang, A.J. Hinz, et al., The biofilmspecific antibiotic resistance gene ndvB is important for expression of ethanol oxidation genes in Pseudomonas aeruginosa biofilms. Journal of bacteriology, 2012, 194(12): 3128-3136.

[42] J.F. Committee, British national formulary (BNF) 66. Vol. 66. Pharmaceutical Press, 2013.

Copyright $₫$ Shaimaa Obaid Hasson. This is an open-access article distributed under the terms of the Creative Commons Attribution License, which permits unrestricted use, distribution, and reproduction in any medium, provided the original author and source are credited. 